|Infection Prevention\&Control risks | Environmental preparedness.

Conclusion These recent exercises have demonstrated the potential applications of simulation to support process improvement. Beyond the Pandemic, we aim to continue to deliver Systems Safety Simulation exercises to help make our clinical systems and spaces safer for patients and the teams who care for them.

\section{THE USE OF A HIGH FIDELITY SIMULATOR TO FACILITATE COACHING IN THE IMPLEMENTATION AND MANUAL TITRATION OF NON-INVASIVE VENTILATION (NIV) DURING A CARDIORESPIRATORY SLEEP STUDY IN A CONTROLLED AND SAFE ENVIRONMENT}

Gabrielle Simpson, Matthew Davies. Great Ormond Street Hospital

\subsection{6/archdischild-2020-gosh. 105}

Introduction The aim of this programme was to pilot a simulation to help develop practitioners' confidence in decision making and implementation of NIV in set scenarios whilst having the support of senior practitioners' in debrief.

Methods Using a high fidelity simulator candidates were exposed to an immersive simulation which accurately obtained physiological data within the sleep recording system as expected in clinical practice. The level of immersion was hugely increased with the utilisation of Simulation Technicians' knowledge in the capabilities of the high fidelity manikin, together with subject matters and experts enabling an authentic environment. Trialling this over a 6 month period was required to create complex paediatric respiratory sleep patterns which are commonly seen in clinical practice when CYP are treated with NIV, such as apnoeas, hypopnoeas and changes in gas exchange parameters including oxygen saturations and carbon dioxide measurements.

Two half day sessions were built around 2-3 scenarios allowing for substantial debrief to identify any human factors or gaps in clinical knowledge. Further, a pre and post confidence survey was conducted.

Results As previously discussed, a pre and post confidence survey was conducted. The questions were focused and specific to match the learning objectives and the needs identified in the initial educational needs analysis. The overall increase in confidence averaged 2.1 and all candidates discussed the direct impact this would have on their clinical practice.

Discussion The use of simulation for paediatric sleep studies with manual titration of ventilation has not been undertaken before in the UK. The pilot study identified the need to progressively increase the complexities of each scenario, whereby candidates feel comfortable to make appropriate clinical decisions in a safe controlled environment. Furthermore, it highlighted a necessity for the manufacturers to develop instant paediatric respiratory pattern and gas exchange alteration packages within the manikin software.

\section{SUPPORT FOR INTERNATIONAL MEDICAL GRADUATES (IMG) IN PANDEMIC AT GREAT ORMOND STREET HOSPITAL (GOSH)}

Ashwin Pandey, Pinki Munot, Daljit Hothi. Great Ormond Street Hospital
Background They are overseas trained doctors. In 2013 GMC declared that $37 \%$ of medical workforce in the UK are overseas trained. GOSH has the highest number of overseas trainee in the UK. In June 2020 we had 116 IMGS. Key challenges faced while settling are knowing the NHS, Governance, communication skills and cultural expectation in the UK. PGME at GOSH works with IMGS to overcome these challenges.

The Pandemic Due to COVID 19 outbreak, IMGs faced many new challenges. With most IMG's living away from their families, major concerns during the pandemic were sickness, social support, personal transport, accommodation, health of family members back home, travel restrictions, financial difficulty, visa renewal and many more. A targeted approach was devised to resolve the day by day emerging unique challenges. IMG support team made assessment of situation at the start of pandemic and existing IMG specific social media platforms were utilized in gathering information and providing customized support. Early recognition of IMGs as vulnerable group and establishing good communication channel by including IMG members in bronze meetings were the major steps that helped us to provide essential support.

Support GOSH provided - arranging accommodation for isolation, care during sickness, supply of food, emotional support for the sick ones, nursery support letters, staff testing, liaison with $\mathrm{HR}$ and pensions, liaising with home department to resolve visa renewal issues, helping in visa extension, training and support to the new recruits, raising the concerns to medical bodies like RCPCH and BMA.

Conclusion It was possible to address these issues in a timely fashion as GOSH had a pre-existing robust IMG support team. Ongoing challenges like travel to see family abroad, quarantines in both countries, impact on training still remains to be addressed and requires continued work towards improving the stay of IMG in the UK.

\section{THE EXTENDED SISTRUNK PROCEDURE FOR THYROGLOSSAL DUCT CYSTS: A REVIEW OF 168 CASES}

${ }^{1}$ Nikita Mehtani, ${ }^{2}$ Claire Frauenfelder, ${ }^{2}$ James Rudd, ${ }^{2}$ Benjamin Hartley. ${ }^{1}$ Imperial College Healthcare NHS Trust; ${ }^{2}$ Great Ormond Street Hospital

\subsection{6/archdischild-2020-gosh.107}

Objectives Thyroglossal duct cysts are the most common paediatric midline neck mass. Recurrence reported in literature following classical Sistrunk's operation remains a problem for $10 \%$ of patients, up-to $20 \%$ if there has been previous surgery.

We have previously published a short series advocating the extended Sistrunk's procedure: comprehensive removal of a block of midline infrahyoid tissue to the level of the thyroid isthmus, incorporating the tract remnant, medial adjacent strap muscles, mid-portion of the hyoid, and superiorly to the submucosal tongue base. We present a large, patient series from a single institution to describe outcomes of this procedure which we perform routinely for thyroglossal duct cyst excision.

Method A retrospective, single centre case series is described, analysing clinical outcomes of patients treated with an extended Sistrunk's procedure between 2003 - 2020.

Results 168 patients underwent an extended Sistrunk's procedure during the study period. 32 patients were referred for 
revision surgery. 146 had histologically confirmed thyroglossal duct cysts. Alternative pathologies were: 16 dermoid cysts, 2 ectopic thyroid tissue foci, 1 scar tissue only, 1 mucus retention pseudocyst, 1 development cyst \& 1 fourth arch anomaly.

26 patients experienced a minor post-operative complication (managed conservatively). 4 patients required further operative intervention: 2 revision surgery and 2 drainage of haematoma. Overall recurrence rate was 1 (1.6\%).

Conclusion We present the largest published case series of patients who have undergone the extended Sistrunk's procedure. We accept the small increase in conservatively managed wound complications (e.g. wound swelling or minor haematomas) and note the very low return to theatre rate. We advocate for use of this technique even for primary cases as the recurrence rate is low with little peri- or post-operative morbidity.

\section{INVESTIGATING THE MODIFIABLE PSYCHOSOCIAL VARIABLES INFLUENCING ACCESS TO AND OUTCOMES AFTER KIDNEY TRANSPLANTATION IN CHILDREN - A STUDY PROTOCOL}

${ }^{1} \mathrm{Ji}$ Soo Kim, ${ }^{1}$ Jo Wray, ${ }^{2}$ Stephen Marks. ${ }^{1}$ Great Ormond Street Hospital for Children NHS Foundation Trust; ${ }^{2}$ Paediatric Nephrology, Great Ormond Street Hospital for Children NHS Foundation Trust

\subsection{6/archdischild-2020-gosh.108}

Introduction Kidney transplantation, compared with dialysis, is often seen as the gold standard in optimising health, reducing mortality and improving quality of life in children with End Stage Kidney Disease (ESKD). We recently surveyed 12 out of 13 UK paediatric nephrology centres on their transplantation plans for all children registered with ESKD. The most commonly cited factors delaying kidney transplantation in these children were: disease-related (36\%), availability of a suitable donor (27\%) and the child's size (20\%). In 19\% of children, psychosocial factors were listed as a barrier. Some factors, including psychosocial, may be modifiable through local or national intervention. To inform future interventions, further study is needed to explore the range and nature of these psychosocial factors.

Aim To investigate the psychosocial factors that influence access to and outcomes of kidney transplantation among children in the UK.

Methods and Analysis This is a prospective multicentre (13 UK paediatric nephrology centres) mixed-methods study with QUAL-QUANT and QUANT-QUAL phases. First, we will use thematic analysis to review interviews conducted with NHS professionals, children with ESKD and their families that explore these psychosocial factors. Next, validated questionnaires that measure these psychosocial factors will be distributed to the wider UK cohort of pre-transplant children with ESKD and their families. They will be followed up to 2 years regardless of whether they do or do not receive a kidney transplant. Clinical data will be prospectively collected from local hospital notes and registry data (UK Renal Registry and NHS Blood \& Transplant). Families with outlier results will be invited for further interview to explore their findings.

\section{9 COCATOO: A COHORT COMPARISON TOOL FOR GOSH CLINICAL RESEARCHERS}

${ }^{1}$ Lydia Briggs, 'William Bryant, 'Mohsin Shah, 'John Booth, 'Richard Issitt, ${ }^{1}$ Anastassia Spiridou, ${ }^{2}$ Neil I Sebire. ${ }^{1} D R I V E$, Great Ormond Street Hospital for Children NHS Foundation Trust; ${ }^{2}$ GOSH DRIVE

10.1136/archdischild-2020-gosh.109

When faced with large amounts of data, it can be a challenging and lengthy process for a researcher to identify key differences and trends between different sets of patients. The development of a reporting tool which can be easily adapted to any speciality and patient selection would be advantageous in highlighting and presenting patient group comparisons in a clinical research environment.

By developing standard, reproducible analytics from the core datasets provided by the Digital Research Environment (DRE) team at GOSH, the researcher is able to identify differences in hospital (hospital admissions, ward stays) and clinical (patient laboratory results, demographics and vital signs) trends between user defined cohort-control patient groups which can accelerate their clinical understanding.

Herein, we introduce COCATOO, a cohort comparison tool that compares and reports distinct sets of patient groups based on defined specifications which are controlled by the researcher. By utilising generalisable analytics developed in $\mathrm{R}$, the tool is able to display a set of predefined exploratory data analytics (EDA) which have the ability to be adapted to any hospital department by incorporating project specific rules.

We present here an example with Chronic Kidney Disease (CKD) comparing stage 5 to stages $1-4$. The output report gives an overview of the disease and highlights significant cohort-control differences via the predefined EDA.

\section{CLINICAL AND OPERATIONAL INSIGHTS: WORKING TO EMBED DATA-DRIVEN PROCESSES AT GOSH}

${ }^{1}$ William Bryant, ${ }^{1}$ Lydia Briggs, ${ }^{1}$ Richard Issitt, ${ }^{1}$ Mohsin Shah, ${ }^{1} J o h n$ Booth, ${ }^{1}$ Anastassia Spiridou, ${ }^{2}$ Neil J Sebire. ${ }^{1} D R I V E$, Great Ormond Street Hospital for Children NHS Foundation Trust; ${ }^{2}$ GOSH DRIVE

\subsection{6/archdischild-2020-gosh.110}

With clinical and operational data for research more easily accessible than ever before through the Digital Research Environment (DRE) in DRIVE, it now is possible to use dataintensive methods and advanced analytics to address many challenges and provide new insights, both clinical and operational. Opportunities for innovation are plentiful and come in many forms, from dashboarding key indicators and trends, to predicting patient needs, forecasting service demand and more. With the expertise and experience gathered within the DRE Team, we are able to leverage key commonalities between projects and focus on particular challenges faced by diverse teams across the organisation.

Herein we introduce our workflow for data extraction, transformation, presentation and analysis and how we can use these to add value for both clinical and operational data use. We also discuss how we can translate requirements and constraints from various teams in order to drive refinements in data presentation and process optimisation. We present two 